Article: Extrapolation of ASDEX Upgrade H-mode discharges to ITER

\title{
Extrapolation of ASDEX Upgrade H-mode discharges to
}

\section{ITER}

\author{
G. Tardini ${ }^{1}$, O. J. W. F. Kardaun ${ }^{1}$, A. G. Peeters ${ }^{2}$, G. V. Pereverzev ${ }^{1}$,
}

A. C. C. Sips ${ }^{1}$, J. Stober ${ }^{1}$, and the ASDEX Upgrade Team ${ }^{1}$,

${ }^{1}$ Max-Planck-Institut für Plasmaphysik, Euratom Association, Boltzmannstrasse

2, D-85748 Garching, Germany

${ }^{2}$ Centre for Fusion, Space and Astrophysics, Physics Department, University of Warwick, CV4 7AL, Coventry UK

\begin{abstract}
In this paper we discuss a procedure to evaluate the fusion performance of ASDEX Upgrade discharges scaled up to ITER. The kinetic profile shape is taken from the measured profiles. Multiplication factors are used to obtain a fixed Greenwald fraction and an ITER normalised thermal pressure like in the corresponding ASDEX Upgrade discharge. The toroidal field and the plasma geometry are taken from the ITER FEAT design (scenario 2), whereas q95 $_{95}$ is taken from the experiment. The confinement time is inferred assuming that the measured H-factor with respect to several existing scaling laws holds also for ITER. While retaining the information contained in the multi-machine databases underlying the different scaling laws, this approach adds profile effects and confinement improvement with respect to the ITER baseline, thus including recent experimental evidence such as the prediction of peaked density profiles in ITER. Under this set of assumptions, of course not unique, we estimate the ITER performance on the basis of a wide database of ASDEX Upgrade H-mode discharges, in terms of fusion power, fusion gain and triple product. According to the three scaling considered, there is finite probability to reach ignition, while more than half of the discharges require less auxiliary power than the one foreseen for ITER. For all scaling laws, high values of the thermal $\beta_{N}$ up to 2.4 are accessible. A sensitivity study gives an estimate of the accuracy of the extrapolation. The impact of different levels of tungsten concentration on the fusion performance is also studied in this paper. This scaling method is used to verify some common 0D figures of merit of ITER's fusion performance.
\end{abstract}




\section{Introduction}

Various scaling laws have been developed to predict the thermal confinement and fusion performance of ITER ELMy H-mode discharges. The well established IPB98 $(\mathrm{y}, 2)$ scaling law [1] features a strong power degradation; as discussed in Reference [1], this implies a negative $\beta$ dependence. More recently other scalings have been proposed with zero or weak $\beta$ dependence, such as the scaling proposed in equation (9) of [2] (here called 'Cordey' scaling) and the Electro-Static Gyrobohm (ESGB) [3] scaling laws, constructed in order to describe experimental observations in several tokamaks [4][5].

Theory-based models have the advantage of being intrinsically dimensionless and can be used as predictive tools as long as they do not have free parameters to be adjusted to the device. However, they are not fully validated on present tokamaks. Although there is a general agreement about the instabilities that dominate core transport, different models yield different predictions for the core and, moreover, they are strongly sensitive to the values of the kinetic profiles on the pedestal top, which are predicted empirically with large uncertainty $[6][7]$.

In order to predict the fusion performance in ITER, in this work we extrapolate ASDEX Upgrade discharges using some information from the empirical scaling laws and adding some features from present experiments. A large profile database $\left(\mathrm{AUG}_{k i n}^{H}\right)$ of 92 well diagnosed $\mathrm{H}$-mode discharges has been setup, including only stationary time intervals. The scaled profiles have been used for ASTRA [8] simulations with ITER geometry, in order to predict the fusion power $\left(P_{f u s}\right)$, the radiated power $\left(P_{r a d}\right)$ as well as the auxiliary power $\left(P_{\text {aux }}\right)$ needed to sustain the prescribed normalized thermal plasma pressure $\left(\beta_{N, t h}\right)$ at the given H-factor. $P_{f u s}$ is a direct output of the profile normalisation. The H-factors determine the confinement and therefore also the fusion gain $Q=P_{\text {fus }} / P_{a u x}$. In addition, the effect of different levels of tungsten concentration is investigated. Zero-dimensional figures of merit discussed in [9] are compared with the simulation results on the $\mathrm{AUG}_{k i n}^{H}$ database. 


\section{Article: Extrapolation of ASDEX Upgrade H-mode discharges to ITER}

\section{Scaling setup and assumptions}

The values of the main plasma parameters of the discharges of the $\mathrm{AUG}_{k i n}^{H}$ database are reported in Table 1. Some assumptions have been made to scale ASDEX Upgrade experiments up to ITER, according

\begin{tabular}{|c|c|c|c|c|c|c|c|c|c|c|c|c|}
\hline & $\mathrm{I}_{p l}$ & $\mathrm{~B}_{t}$ & $\mathrm{q}_{95}$ & $l i$ & $\frac{\bar{n}_{e}}{n_{G W}}$ & $\frac{n_{e}(0)}{<n_{e}>}$ & $T_{i}(0)$ & $\beta_{N}$ & $\beta_{N, t h}$ & $\mathrm{H}_{98}$ & $\mathrm{H}_{C o r}$ & $\mathrm{H}_{E S G B}$ \\
\hline Aver. & 0.985 & 2.197 & 4.055 & 0.986 & 0.473 & 1.582 & 5.429 & 2.327 & 1.844 & 1.159 & 0.862 & 0.840 \\
Max. & 1.202 & 2.796 & 6.117 & 1.109 & 0.883 & 2.261 & 10.378 & 3.492 & 2.766 & 1.511 & 1.415 & 1.381 \\
Min. & 0.600 & 1.389 & 3.054 & 0.822 & 0.323 & 1.123 & 1.788 & 1.445 & 1.040 & 0.740 & 0.665 & 0.645 \\
\hline
\end{tabular}

Table 1. Main plasma parameters of the selected ASDEX Upgrade discharges. For each parameter, the maximum, minimum and mean values over the database $\mathrm{AUG}_{k i n}^{H}$ are reported.

to a method proposed by Luce et al.[10] and applied also to DIII-D data. The toroidal field, the equilibrium boundary and the impurities' concentration are taken from the ITER-FEAT design [1]. The parameter q95 is changed with respect to the ITER target to be the measured value in the selected ASDEX Upgrade discharges. The plasma current is hence determined. The experimental density profile shape is kept, while its radially constant multiplying factor is chosen such that the line-average density is a given fraction of the Greenwald limit in the ITER plasma, the reference being the ITER target value $85 \%$. Electron and ion temperature profiles are assumed to be equal in ITER, since the heat exchange among the species is predicted to be fast compared to the energy confinement time $\tau_{E}$. Although fusion $\alpha$ particles heat mainly electrons, the rapid heat exchange between ions and electrons makes that heat transport is carried mainly by the channel with the higher anomalous transport under the experimental circumstances. One can roughly assume the channel with higher central temperature to carry most of the heat flux in the core plasma. So we choose the ITER temperature profiles to be proportional to the ASDEX Upgrade temperature profile with the highest central value. This assumption plays actually a minor role, because the temperature gradient lengths for ions and electrons are quite close to each other in the $\mathrm{AUG}_{\text {kin }}^{H}$ database. The scaling factor for the temperature profiles is determined by setting $\beta_{N, t h}$ to be a given fraction of the ASDEX Upgrade value, obtained integrating the measured kinetic profiles. The default value is $\beta_{N, t h}=\beta_{N, t h}{ }^{A U G}$. The deuterium and tritium concentrations are assumed to be equal. The impurity concentration is taken from the ITER design: Be $2 \%$, Ar 0.12 \% [7] and He $4.3 \%$, which is within the range considered in [7]. As a result, the volume averaged $\mathrm{Z}_{e f f}$ is approximately 


\section{G. Tardini et al.}

1.65 in all simulations of tungsten-free plasmas. A scan of the tungsten concentration is performed in addition to the mentioned impurities. The radiation model from [11] is used. Finally, we assume the confinement time of the ITER discharge to be $\tau_{E, j} \times H_{j}, H_{j}$ being the measured value of the ASDEX Upgrade discharge and $j=1,2,3$ the scaling law label. The auxiliary power required for the scaled discharge is then determined and can be compared to the envisaged heating power in ITER. When evaluating the fusion gains we neglect for simplicity the correction to the total power arising from charge exchange processes and orbit losses in the scaling law. Beam-target fusion is not included either, which is expected to be a negligible heat source in ITER due to the very low injection rate of the highly energetic beam ions and due to the large device size.

\section{Fusion power}

In Fig. 1, the quantity $\beta_{N, t h}$ of the ASDEX Upgrade discharges is plotted versus the total measured $\beta_{N}$. It is obtained by integrating the kinetic profiles, which are a fit of the available diagnostics for a certain quantity. The parameter $\beta_{N, t h}$ ranges between 1 and 3 , whereas $\beta_{N}$ is higher on average by approximately $20 \%$. Of course, the fast ions contribution to the total $\beta_{N}$ can vary from discharge to discharge, depending for instance on the beam power and on the plasma density. The scattering in Fig. 1 is, then, partly explained by physics effects, while experimental uncertainties of the kinetic profiles' measurements also play a role.

In Fig. 2 we show the performance in the case without tungsten (diamonds) compared to the worst case with a volume-averaged concentration $\left\langle n_{W} / n_{e}\right\rangle=10^{-4}$ (stars), the maximum tolerable for ITER [12]. The profile peaking is assumed for simplicity to be the same as the electron density peaking. Fig. 2 (a) shows $P_{f u s}$ as a function of $\beta_{N, t h}^{2} / q_{95}^{2}$, which represent the scaling of the fusion power at fixed magnetic field in the approximation $\mathrm{P}_{f u s} \propto n^{2} T_{i}^{2}$. As expected the effect of fuel dilution by tungsten is negligible. $P_{\text {rad }}$ is calculated in ASTRA as the sum of the bremsstrahlung, the synchrotron radiation and the line radiations of Ar, Be and W. In Fig. 2 (b) a substantial increase of $P_{\text {rad }}$ with tungsten is observed, by an amount comparable to the total auxiliary heating capability of ITER, $73 \mathrm{MW}$. As the temperature profiles are fixed, any increase of $P_{\text {rad }}$ has to be compensated by increasing $P_{a u x}$, and in case of central radiation this is even too optimistic. It has to be noted, however, that $\mathrm{Ar}$ is foreseen in ITER as a radiation actuator, so one might just reduce its concentration in case of high 
Article: Extrapolation of ASDEX Upgrade H-mode discharges to ITER

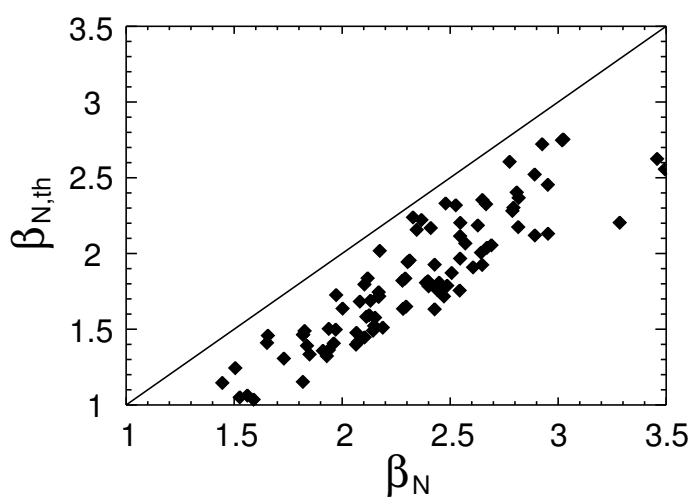

Figure 1. Normalised plasma pressure from the experimental kinetic profiles as a function of the measured total $\beta_{N}$ in the $\mathrm{AUG}_{k i n}^{H}$ database.
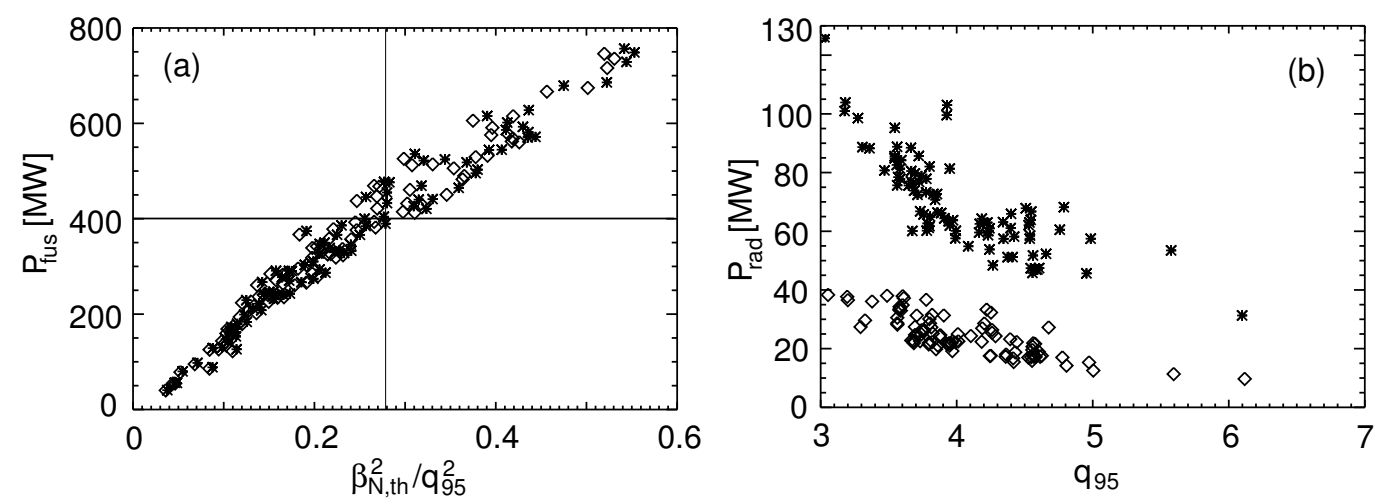

Figure 2. (a) $P_{f u s}$ in an ITER-sized device as a function of $\beta_{N, t h}^{2} / q_{95}^{2}$ without tungsten (diamonds) or with $<n_{W} / n_{E}>=$ $10^{-4}$ (stars). The lines mark the ITER operation point. (b) $P_{r a d}$ versus q95 with different tungsten content in an ITERsized device. Symbols as in (a).

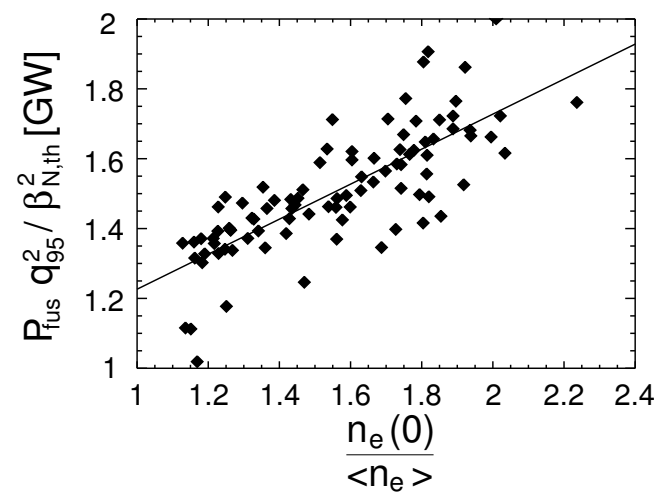

Figure 3. Ratio of $P_{f u s}$ to $\beta_{N, t h}^{2} / q_{95}^{2}$ as a function of the density peaking. 


\section{G. Tardini et al.}

$P_{\text {rad }}$ due to tungsten. In the following sections we consider the case without tungsten. Since the profile shape information plays a role only for $P_{\text {fus }}$, from the almost linear behaviour in Fig. 2 (a) one can conclude that an appropriate 0D scaling is an acceptable approximation of the fusion performance of the scaled discharges. The deviation from a linear dependence of $P_{f u s}$ on $\beta_{N, t h}^{2} / q_{95}^{2}$ is illustrated in Fig. 3. There is a positive correlation between the performance improvement and the density peaking. Some scattering remains, most likely due to the degradation of $P_{f u s}$ at higher temperatures for a given $\beta_{N, t h}$.

\section{Fusion gain}

In Fig. 4 we plot the range of the H-factors and of $\beta_{N, t h}$ in our database. The parameter q95 ranges between

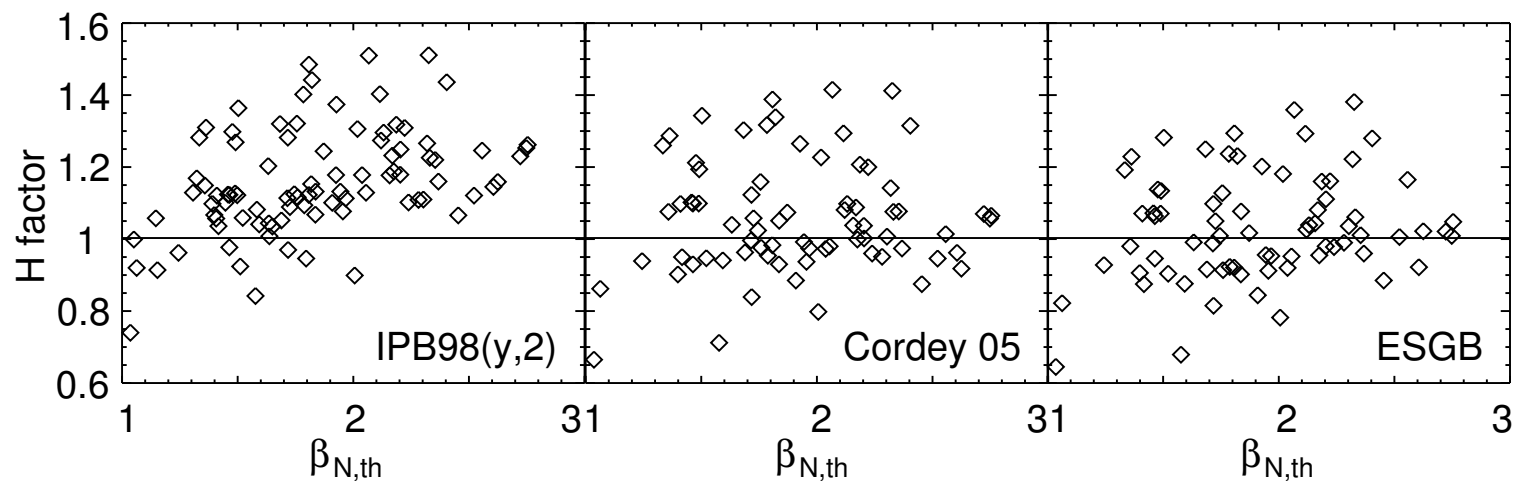

Figure 4. H-factors as a function of $\beta_{N, t h}$ for the IPB98(y,2) (a) Cordey 05 (b) and ESGB (c) scaling laws. The line $\operatorname{marks} \mathrm{H}=1$

3 and 5, except two discharges featuring q95 $_{95}$ around 6 . The experimental H-factors with respect to the empirical the scaling laws [1][2][3] are kept as confinement improvement factors for ITER. As a result, one obtains the required $P_{\text {heat }}$ and hence $P_{\text {aux }}$ as $P_{\text {aux }} \approx P_{\text {heat }}-P_{\alpha}-P_{\text {Ohm }}$. In Fig. 5 the ITER $P_{a u x}$ thus predicted are plotted for the three scaling laws. The two horizontal lines mark $P_{a u x}=0$ and $P_{a u x}=73 \mathrm{MW}$, which corresponds to the scheduled auxiliary heating power in ITER. 'Negative' auxiliary power in Fig. 5 means ignition $(\mathrm{Q}=$ $\infty)$, power larger than $73 \mathrm{MW}$ means that such a $\beta_{N, t h}$ value is not accessible for the given H-factor, given the ITER scheduled auxiliary power. Different symbols are used for extrapolated discharges with fusion power below $400 \mathrm{MW}$ (stars) and above (diamonds), respectively. This symbol code is used for the remainder of this paper. There is a rather clear separation around $\beta_{N, t h}=1.8-2$, which appears in all Figures [5-11]. The most optimistic prediction is obtained with the ESGB scaling, with $26 \%$ of the discharges igniting and $88 \%$ within 


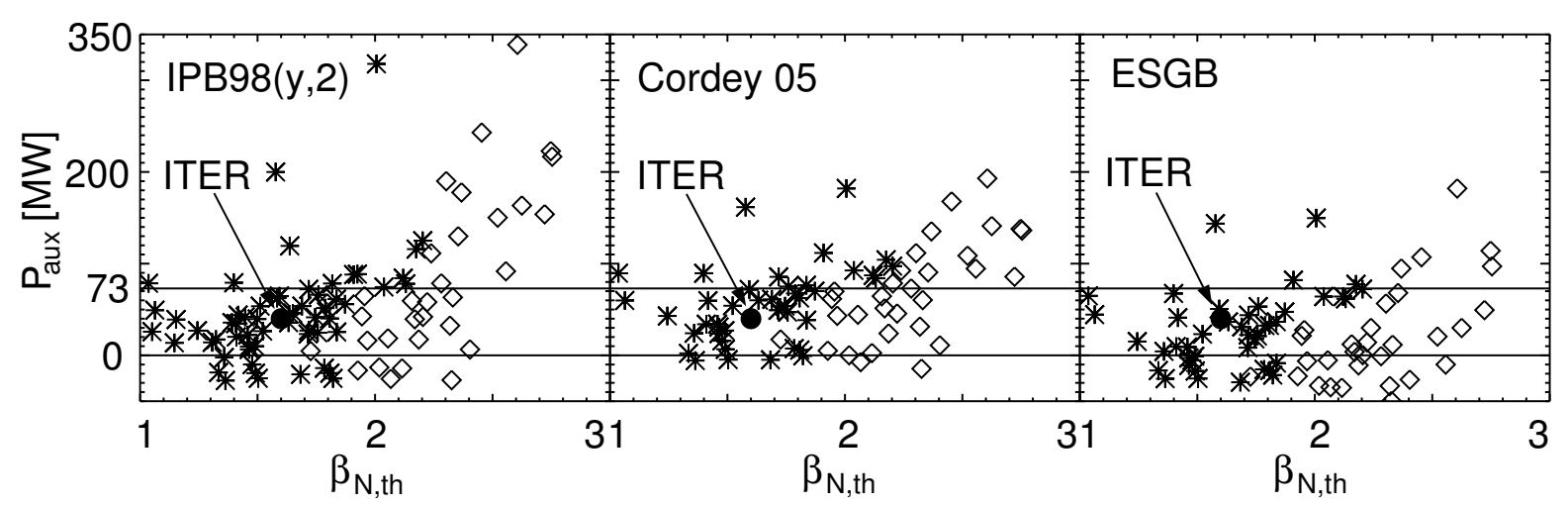

Figure 5. Needed $P_{a u x}$ versus $\beta_{N, t h}$ for the IPB98(y,2) (a) Cordey 05 (b) and ESGB (c) scaling laws. The lines marks $P_{a u x}=0$ (ignition) and $P_{a u x}=73 \mathrm{MW}$ (ITER's scheduled $P_{a u x}$ ). Different symbols refer to fusion power below (stars) and above (diamonds) $400 \mathrm{MW}$. The full circle represents the operational point for the reference ITER scenario 2 [13].

ITER's auxiliary power. The Cordey scaling predicts similar results, with slightly smaller fusion gain. At high $\beta_{N, t h}$ the most conservative prediction is given by the IPB98 $(y, 2)$ scaling, owing to its strong $\beta$ degradation. Nevertheless, high $\beta_{N, t h}$ values up to 2.5 are accessible when we scale up ASDEX Upgrade discharges with the highest $\mathrm{H}$-factor. Ignition is obtained for $16 \%$ of the extrapolated discharges according to the IPB98(y,2) scaling.

\section{Sensitivity study}

We have changed some of our assumptions to verify how critical the extrapolation to ITER is, varying the main parameters $\beta_{N, t h}, H$ and $\bar{n}_{e} / n_{G W}$. The performance of the $\mathrm{AUG}_{k i n}^{H}$ database following the modified assumptions are summarised in Table 2 . In the Figures 6 and $7 \beta_{N, t h}$ has been reduced and increased by $20 \%$, respectively. The IPB98(y,2) scaling is most affected, as expected, because of its stronger power dependence. A $20 \%$ reduction in $\beta_{N, t h}$ results in the almost overall sustainability of the scaled discharges with the ITER heating capability, for all three scalings. Also for the IPB98 $(y, 2)$ scaling the ignition condition is achieved for about $25 \%$ of the discharges. Lower $\beta_{N, t h}$ values are favourable for the fusion triple product for scaling laws with a negative $\beta$ dependence, like the $\operatorname{IPB} 98(\mathrm{y}, 2)$ scaling. The opposite behaviour is observed if $\beta_{N, t h}$ is increased from the reference ASDEX Upgrade value: the IPB98(y,2) scaling predicts almost no ignition and moreover most of the discharges are not sustainable with the presently foreseen ITER auxiliary power, as shown in Fig. 7 . Multiplying $\beta_{N, t h}$ by 0.8 or 1.2 has the effect of zooming the low or high $\beta_{N, t h}$ corner of Fig. 


\section{G. Tardini et al.}

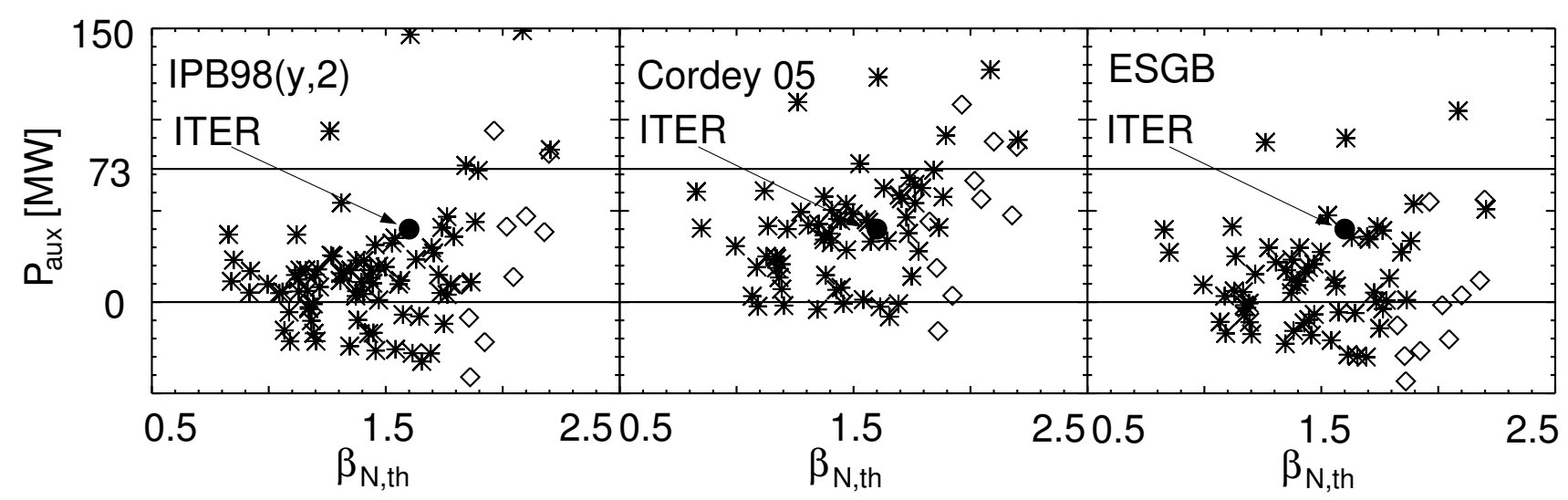

Figure 6. Auxiliary power as a function of $\beta_{N, t h}$ for the scaled discharges, assuming $\beta_{N, t h}{ }^{I T E R}=0.8 \beta_{N, t h}{ }^{A U G}$.

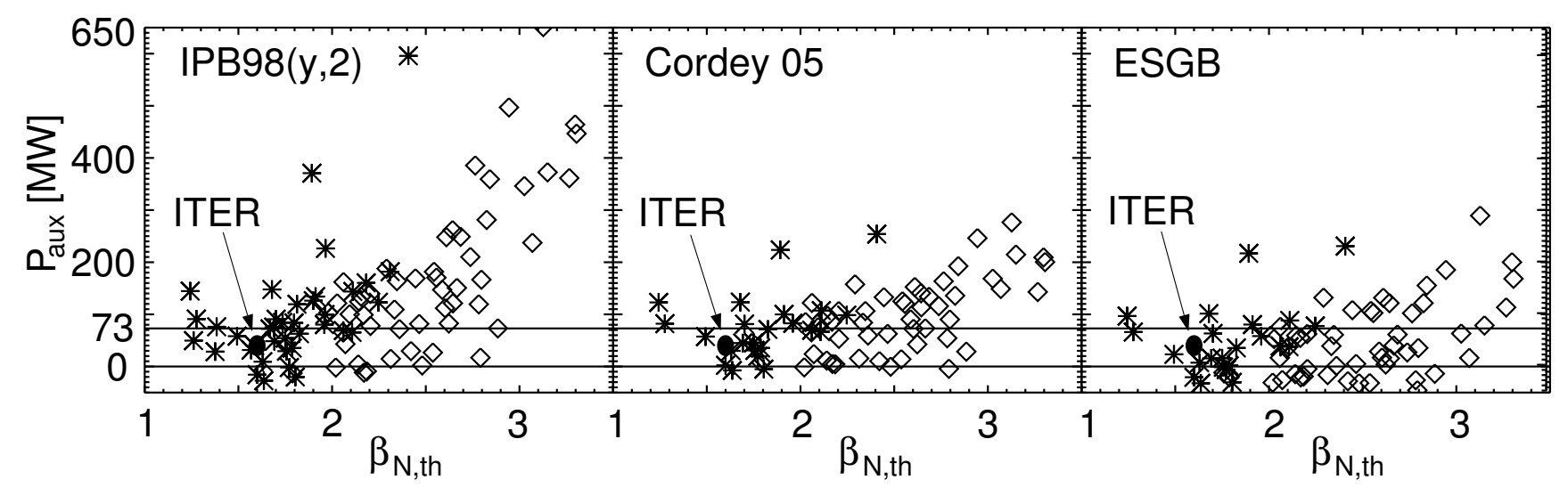

Figure 7. Auxiliary power as a function of $\beta_{N, t h}$ for the scaled discharges, assuming $\beta_{N, t h}{ }^{I T E R}=1.2 \beta_{N, t h}{ }^{A U G}$.

5, respectively. The small amount of points with fusion power above $400 \mathrm{MW}$ (diamonds) in Fig. 6 confirms that the improvement of the fusion gain at lower $\beta_{N, t h}$ happens at cost of a reduced fusion power, as the latter depends quadratically on $\beta_{N, t h}$.

The same exercise for the H-factors is plotted in Fig. 8 and Fig. 9. If the confinement factors are lower by 20 $\%$ with respect to the ASDEX Upgrade discharges, the picture changes quite drastically: ignition is precluded and most of the scaled discharges require far more heating than it will be available in ITER. Conversely, a systematic increase of all $\mathrm{H}$-factors by $20 \%$ leads to an optimistic picture where nearly all ASDEX Upgrade $\beta_{N, t h}$ values can be in fact produced and almost $50 \%$ of the scaled discharges ignite, except for the Cordey scaling, which predicts a lower ignition rate. If the $\mathrm{H}$-factors and the $\beta_{N, t h}$ values from ASDEX Upgrade are kept, instead, it is useful to investigate the effect of the Greenwald fraction. The fusion power is, in fact, roughly quadratic in $T_{i}$ only over a narrow (but relevant) interval of $T_{i}$ [14]. Moreover, the scaling laws [1] [2] [3] have 


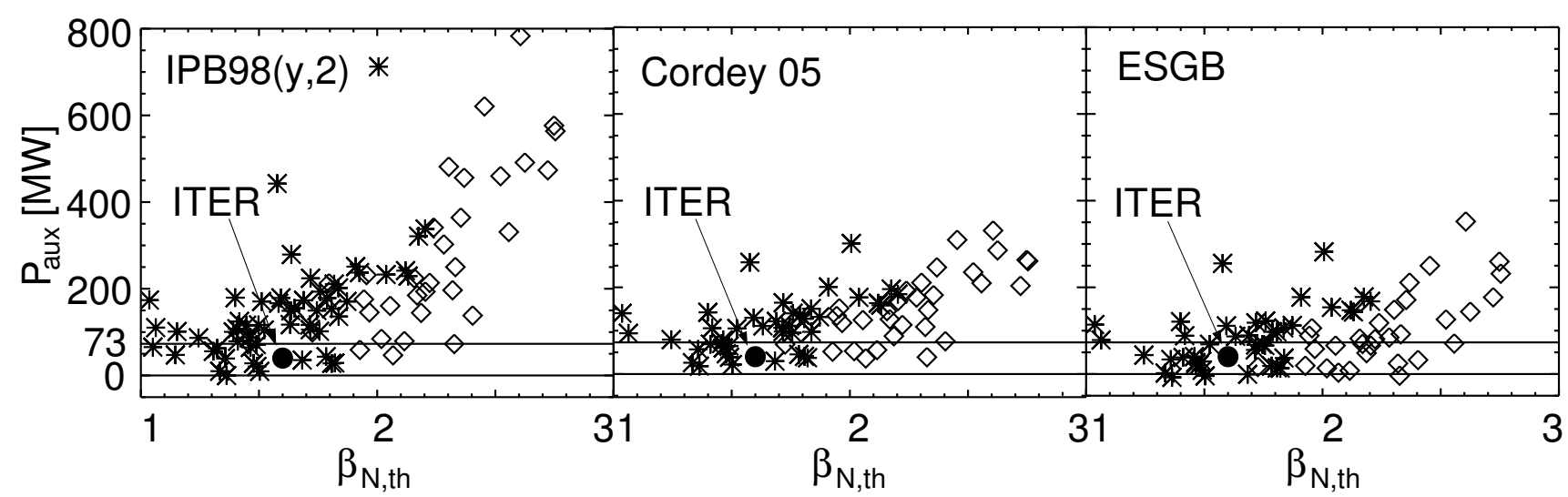

Figure 8. Auxiliary power as a function of $\beta_{N, t h}$ for the scaled discharges, assuming $H^{I T E R}=0.8 H^{A U G}$.

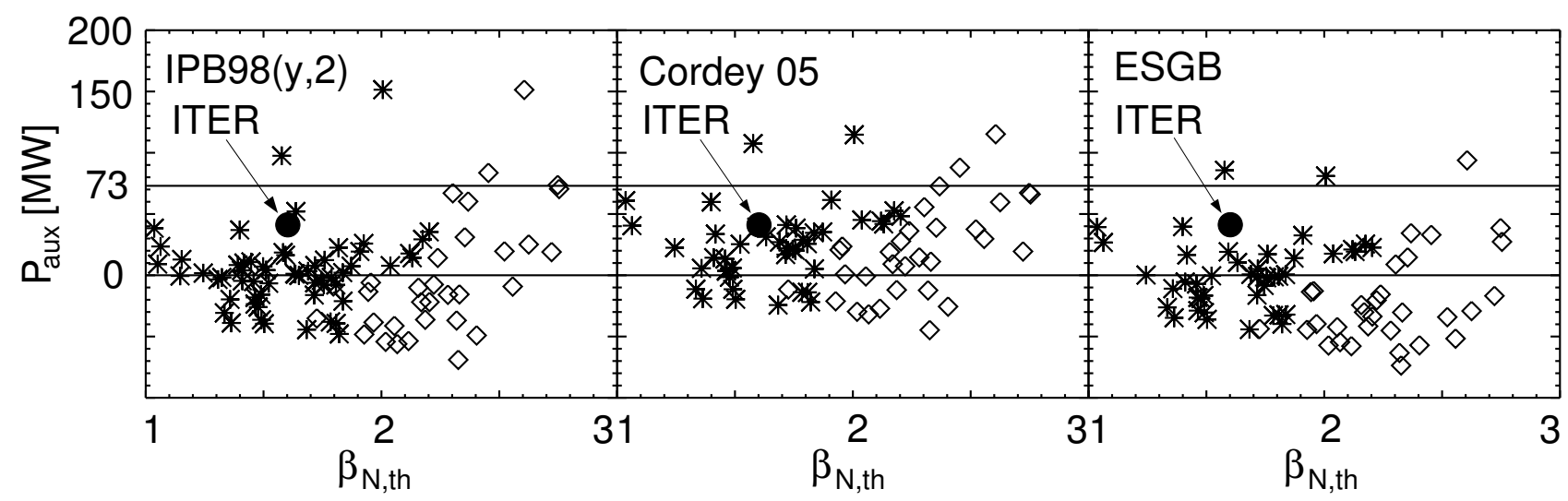

Figure 9. Auxiliary power as a function of $\beta_{N, t h}$ for the scaled discharges, assuming $H^{I T E R}=1.2 H^{A U G}$.

an explicit dependence on the plasma density, each law with a different power. In Fig. 10 it is assumed that $\bar{n}_{e} / n_{G W}=0.7[15]$. Although the effect is not dramatic, it is sizable: ignition is not predicted anymore for the Cordey scaling, whilst for all scaling half of the scaled discharges are not feasible at all, because $P_{\text {aux }}>73 M W$. In the extreme case of a Greenwald fraction $\bar{n}_{e} / n_{G W}=0.95$, instead, ignition becomes a concrete prospect and the ITER auxiliary heating allows to reach the range of $\beta_{N, t h}$ values featured by nearly all ASDEX Upgrade discharges included in our database (see Fig. 11). In summary, a strong sensitivity is found on the key parameters $\beta_{N, t h}, H$ and $\bar{n}_{e} / n_{G W}$, as Table 2 shows. This gives a measure of the accuracy of our prediction when using the reference set of assumptions, since quite different predictions can be achieved varying these parameters within realistic intervals. A quantitative dependence on the three parameters according to the three scaling laws is derived in the following Section, neglecting the effects of the profile shape. 


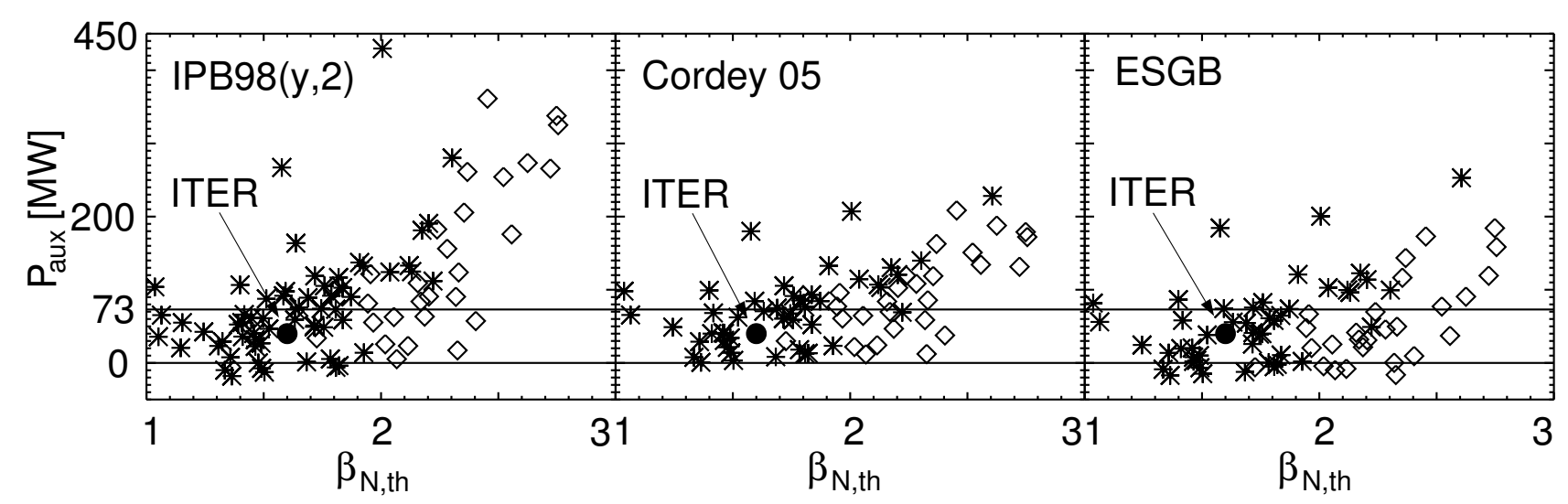

Figure 10. Auxiliary power as a function of $\beta_{N, t h}$ for the scaled discharges, assuming $\bar{n}_{e} / n_{G W}=0.7$ for the ITER plasma.

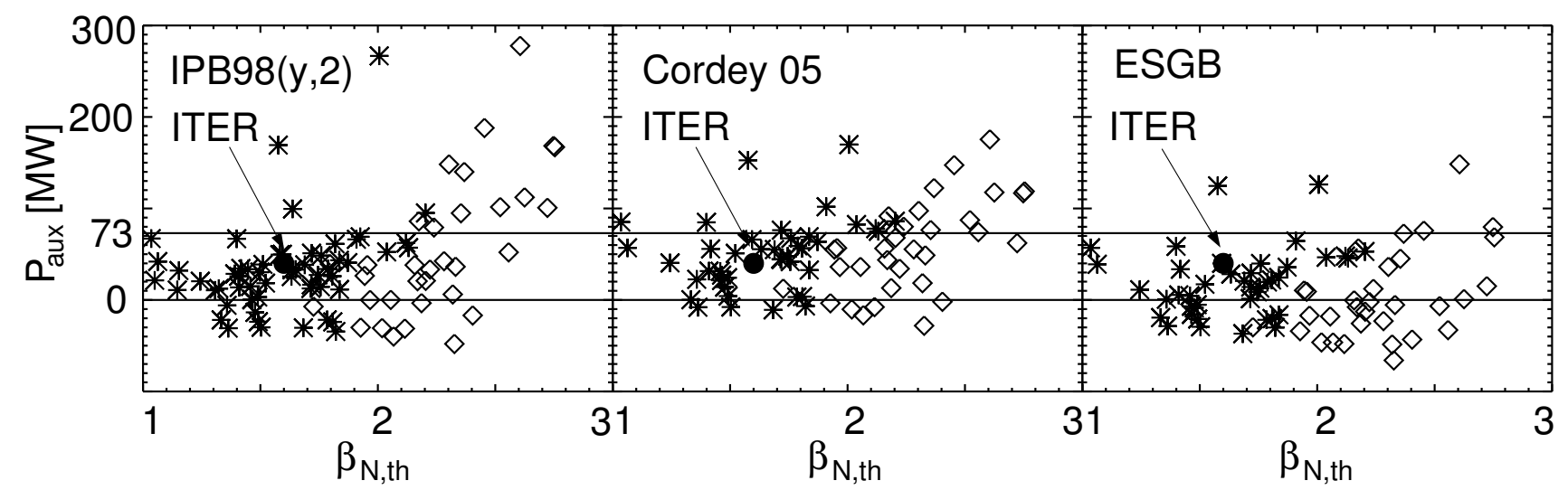

Figure 11. Auxiliary power as a function of $\beta_{N, t h}$ for the scaled discharges, assuming $\bar{n}_{e} / n_{G W}=0.95$ for the ITER plasma.

\section{Verification of figures of merit for fusion performance}

The quantity $G=P_{\text {fus }} /\left(5 P_{\text {heat }}\right)$ is often used in the literature as a figure of merit of the fusion efficiency. In fact, $G \propto n^{2}<\sigma v>\tau_{E} /(n T)$ can be simplified to $G \propto n T \tau_{E}$ because in the relevant temperature range one can roughly approximate $<\sigma v>\propto T^{2}$. An improved formula, $<\sigma v>\propto T^{6.1-1.8 \log T}$ (for $3 \leq T \leq 30 \mathrm{keV}$ ), and hence $\left\langle\sigma v>\propto T^{2.5}\right.$ for $T \approx 10 \mathrm{keV}$, was derived in [14]. Introducing the usual definition $Q=P_{\text {fus }} / P_{a u x}$ one can relate the two quantities: $G=Q /(Q+5)$. In particular, $G=0$ means $P_{\text {fus }}=0$, whereas $G=1$ means ignition. As pointed out in [9], however, finite power degradation in the scaling laws has to be considered when choosing the correct scaling of $G$ with respect to $H, \beta_{N, t h}$ and $q_{95}$. Our approach assumes constant $B$, geometry, 
Article: Extrapolation of ASDEX Upgrade H-mode discharges to ITER

\begin{tabular}{|l|cc|cc|cc|}
\hline & \multicolumn{2}{|c|}{ IPB98(y,2) } & \multicolumn{2}{|c|}{ Cordey } & \multicolumn{2}{|c|}{ ESGB } \\
Assumption & Ignition & $\mathrm{P}_{\text {aux }}<73 \mathrm{MW}$ & Ignition & $\mathrm{P}_{\text {aux }}<73 \mathrm{MW}$ & Ignition & $\mathrm{P}_{\text {aux }}<73 \mathrm{MW}$ \\
\hline Default & $16 \%$ & $71 \%$ & $8 \%$ & $64 \%$ & $35 \%$ & $88 \%$ \\
\hline$\beta_{N, t h}=0.8 \beta_{N, t h}^{A U G}$ & $26 \%$ & $92 \%$ & $11 \%$ & $88 \%$ & $38 \%$ & $96 \%$ \\
\hline$\beta_{N, t h}=1.2 \beta_{N, t h}^{A U G}$ & $9 \%$ & $35 \%$ & $7 \%$ & $45 \%$ & $27 \%$ & $69 \%$ \\
\hline$H=0.8 H^{A U G}$ & $1 \%$ & $21 \%$ & $0 \%$ & $27 \%$ & $5 \%$ & $49 \%$ \\
\hline$H=1.2 H^{A U G}$ & $53 \%$ & $95 \%$ & $26 \%$ & $95 \%$ & $64 \%$ & $96 \%$ \\
\hline $\bar{n}_{e} / n_{G W}=0.7$ & $7 \%$ & $47 \%$ & $0 \%$ & $51 \%$ & $16 \%$ & $68 \%$ \\
\hline $\bar{n}_{e} / n_{G W}=0.95$ & $20 \%$ & $82 \%$ & $14 \%$ & $70 \%$ & $43 \%$ & $93 \%$ \\
\hline
\end{tabular}

Table 2. ITER performance of the database $\mathrm{AUG}_{k i n}^{H}$ modifying the baseline assumptions of $\beta_{N, t h}, H$-factor and $\bar{n}_{e} / n_{G W}$. Only one parameter is varied at a time.

ion mass and $\bar{n}_{e} / n_{G W}$. The $G$ scaling derived in [9] reads:

$G=C H^{\frac{1}{1+\alpha_{p}}} \beta_{N, t h}^{2-\frac{1}{1+\alpha_{p}}} q_{95}^{-1-\frac{\alpha_{p}+\alpha_{I}+\alpha_{n}}{1+\alpha_{p}}}$

where $\alpha$ 's are the scaling law's exponents for power $\left(\alpha_{p}\right)$, current $\left(\alpha_{I}\right)$ and density $\left(\alpha_{n}\right)$ dependence. The

\begin{tabular}{|l|c|c|c|c|c|c|}
\hline & $\alpha_{p}$ & $\alpha_{I}$ & $\alpha_{n}$ & $\exp _{\beta_{n}}$ & $\exp _{q_{95}}$ & $\exp _{H}$ \\
\hline IPB98 $(y, 2)$ & -0.69 & 0.93 & 0.41 & -1.23 & -3.10 & 3.23 \\
\hline Cordey & -0.45 & 0.85 & 0.26 & 0.18 & -2.20 & 1.82 \\
\hline ESGB & -0.55 & 0.83 & 0.49 & -0.22 & -2.71 & 2.22 \\
\hline
\end{tabular}

Table 3. Exponents of the power, current and density dependences of the different scaling laws, and the resulting exponents for the scaling of $\mathrm{G}$ as a function of $\beta_{N}, \mathrm{q}_{95}$ and $H$ at constant Greenwald fraction.

coefficient $C$ for each scaling law is determined at a given ITER reference scenario. Fig. 12 shows a good agreement between the $Q /(Q+5)$ of the ASTRA simulations of the scaled discharges and the 0D scaling for $G$ given by equation 1 . Taking, for instance, the $0 \mathrm{D}$ G-scaling based on the assumption of a constant absolute density, i.e. equation 1 without $\alpha_{n}$, the data alignment becomes significantly worse (see Fig. 13). This is consistent with the fact that in the simulations we have chosen to keep the Greenwald fraction fixed, not the absolute plasma density. The Cordey scaling is less affected, because the q95 $_{95}$ dependence is not significantly changed with respect to the case with constant Greenwald density. For the parameter $H \beta_{N, t h} / q_{95}^{2}$, quite 


\section{G. Tardini et al.}

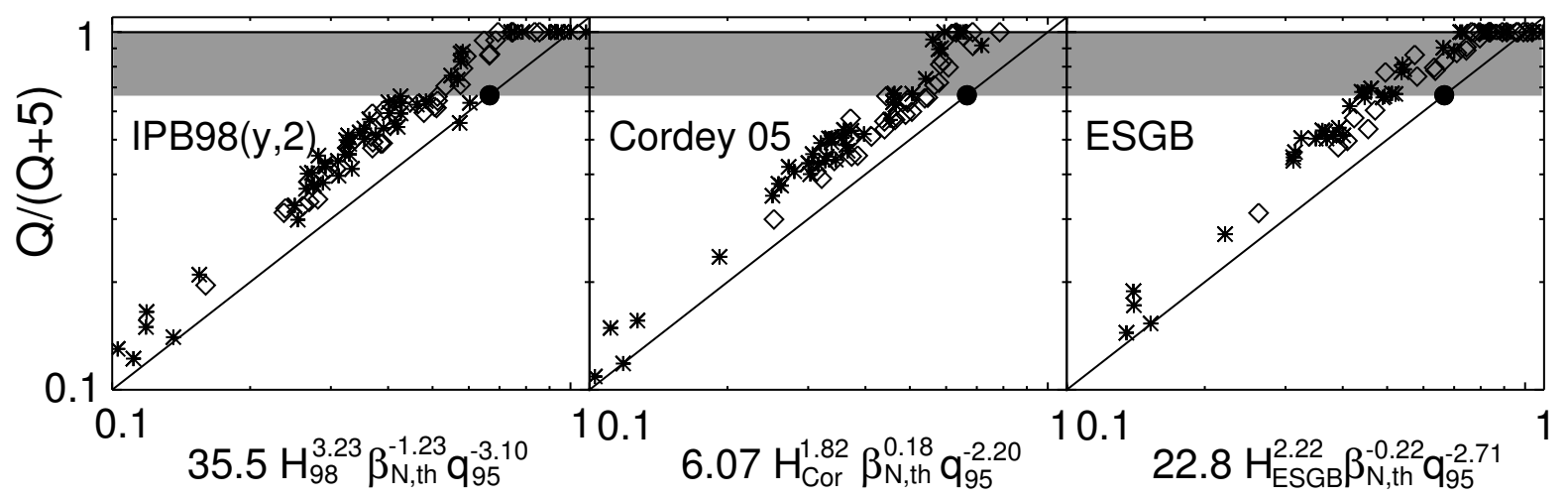

Figure 12. G from ASTRA simulations as a function of the $0 \mathrm{D}$ figure of merit for $\mathrm{G}$ at constant $\bar{n}_{e} / n_{G W}=0.85$. In the shaded region $\mathrm{Q} \geq 10$. $\mathrm{Q} /(\mathrm{Q}+5)=1$ corresponds to ignition.

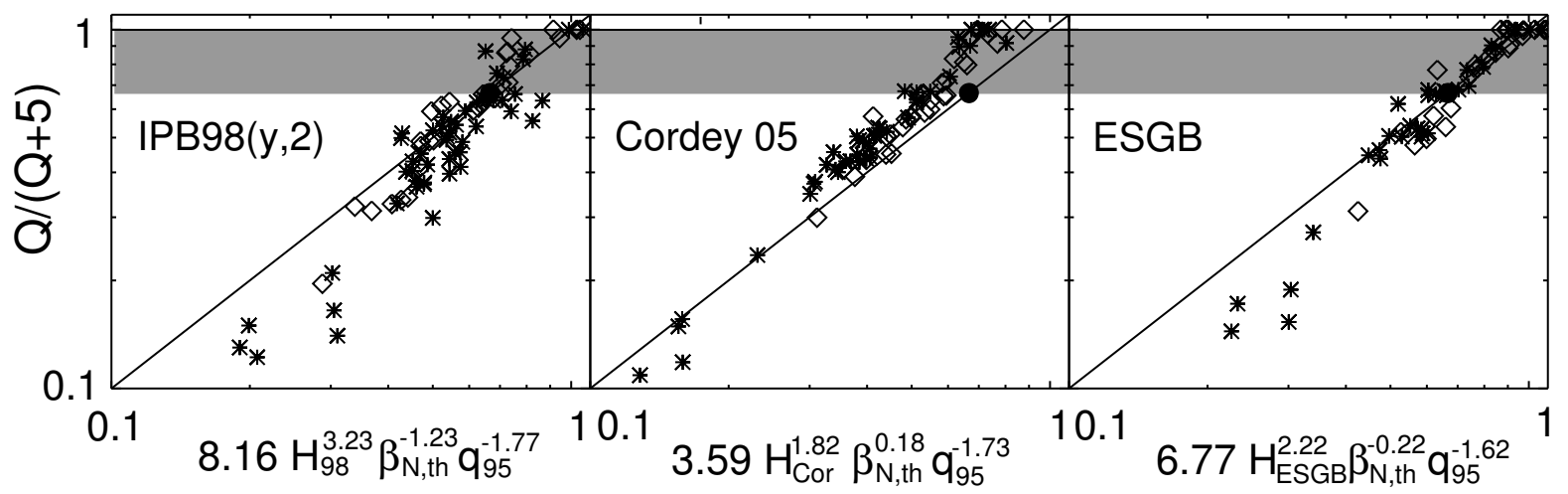

Figure 13. G from ASTRA simulations as a function of the $0 \mathrm{D}$ figure of merit for $\mathrm{G}$ at constant $n_{e}$. Note that the exponents of $\mathrm{q}_{95}$ are different than in Fig. 12. They are derived in [9].

common in the literature, almost no alignment is observed for the IPB98(y,2) scaling, as Fig. 14 highlights. Even for the ESGB and Cordey scaling laws, which have a weaker power degradation, the scattering increases significantly compared to Fig. 12. In fact, the parameter $H \beta_{N, t h} / q_{95}^{2}$ is not equivalent to the triple product, unless both conditions $\alpha_{p}=0$ and $\alpha_{I}+\alpha_{n}=1$ are fulfilled simultaneously, which is not the case for the scaling laws considered here. Interestingly, in Fig. 14 the points with low (stars) and high (diamonds) fusion power are perfectly separated. This means that $H \beta_{N, t h} / q_{95}^{2}$ is systematically overestimated for high power discharges, therefore it should not be used as an indicator of the triple product in tokamaks operating at variable auxiliary power, let alone for comparing the discharge performance from different tokamaks.

A high fusion gain like $\mathrm{Q}=10$ is, however, not the only goal of ITER, which is supposed to deliver also a significant amount of fusion power in order to provide a convincing test on the road towards a prototype fusion 
Article: Extrapolation of ASDEX Upgrade H-mode discharges to ITER

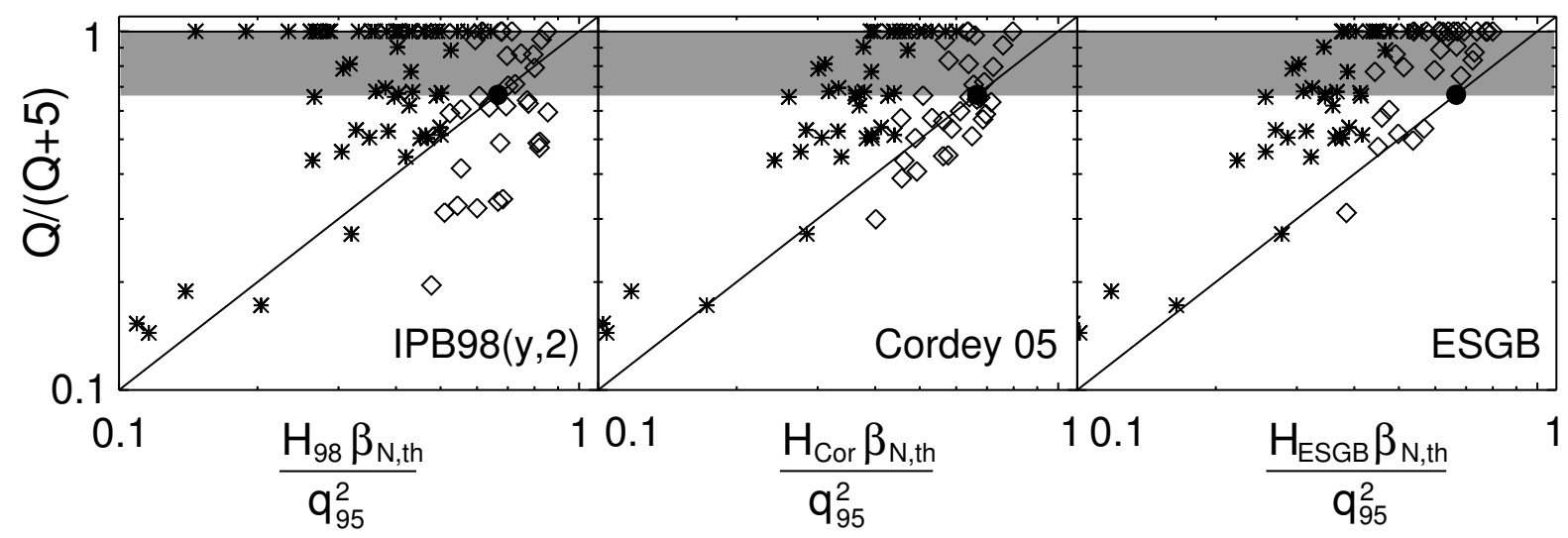

Figure 14. G from ASTRA simulations as a function of the parameter $H \beta_{N, t h} / q_{95}^{2}$. Points with a high fusion power (diamonds) exhibit a systematic overestimate of $H \beta_{N, t h} / q_{95}^{2}$.

reactor. Moreover, the scaling laws used here are valid only for H-mode plasmas, so one has to make sure that the H-mode is reached. This provides a constraint to the reduction of the auxiliary power, because the total heating must exceed the threshold power [16]. Therefore, one might prefer to choose a parameter which is a compromise between an efficient fusion gain and high fusion power in ITER, such as $H \beta_{N, t h} / q_{95}^{2}$. We note that there is no generally valid, unique definition of "figure of merit of fusion performance". An appropriate choice depends on the kind of investigation, provided the meaning of the figure of merit is defined quantitatively.

Finally, Fig. 12 shows that the 0D scaling proposed in [9] and summarised in Table 3 is accurate enough to predict the figure of merit of ignition, with moderate corrections associated with the profile shape and the varying dependence of the fusion cross section on $T_{i}$, which is a function of $T_{i}$ itself. The ITER operational point is systematically below the scaled ASDEX Upgrade discharges, possibly due to the zero density peaking assumed in the reference simulations.

\section{Conclusions}

A method for the extrapolation of present day tokamak discharges to ITER using measured profiles is applied to a database of ASDEX Upgrade H-mode discharges. Different scaling laws are used and the discharge performance is evaluated according to the improvement factors of the ASDEX Upgrade experiments. As a result of our assumptions on $\beta_{N, t h}$ and $\bar{n}_{e} / n_{G W}$, the large majority of the discharges are sustainable with the 


\section{G. Tardini et al.}

heating power scheduled for ITER. The Cordey and the ESGB scaling laws have, of course, no trend to worse triple product (or higher auxiliary power) with increasing $\beta_{N, t h}$, ESGB being the most optimistic: $35 \%$ of the extrapolated discharges fulfills the ignition condition. Even the more pessimistic IPB98(y,2) scaling predicts $\beta_{N, t h}$ values up to 2.5 to be achievable with the $\mathrm{H}$-factors as contained in our database, ignition being reached by $16 \%$ of the discharges.

The effect of the tungsten concentration is shown to be negligible in terms of fuel dilution for the achieved fusion power. However, at the maximum tolerable value of $n_{W} / n_{e}=10^{-4}$ the radiated power becomes comparable to the auxiliary power of ITER, thus reducing significantly the fusion gain Q.

The database of 92 discharges already displays a wide range in $\beta_{N}$ and H-factors. Nevertheless, we performed a sensitivity study scanning the $\mathrm{H}$-factors, $\beta_{N, t h}$ with respect to the ASDEX Upgrade value and the imposed Greenwald fraction. The parameter $\beta_{N, t h}$ has a drastic impact on the IPB98(y,2) scaling: although the fusion power increases quadratically with $\beta_{N, t h}$, the auxiliary power required to sustain such a discharge grows by factors with a $20 \%$ increase in $\beta_{N, t h}$. The Cordey and ESGB scaling are, as expected, less affected, but still the required auxiliary power increases: $55 \%$ and $31 \%$ of the discharges are beyond ITER's reference heating capability of $73 \mathrm{MW}$, respectively. A change in $20 \%$ in the H-factors leads to extreme variations for all the scaling laws considered. Increasing $\mathrm{H}$, almost all discharges are achievable and roughly half of them ignite. Reducing $\mathrm{H}$, the vast majority of the discharges exceeds the scheduled heating power and is, therefore, not accessible. Lower Greenwald fraction are, of course, unfavourable, but going from $\bar{n}_{e} / n_{G W}=0.7$ to $\bar{n}_{e} / n_{G W}=$ 0.95 the effect is moderate.

A 0D treatment allows to compute the exact dependence of the triple product, or equivalently $\mathrm{Q} /(\mathrm{Q}+5)$, on the $\mathrm{H}$-factor, $\beta_{N, t h}$ and $q_{95}$, the only free parameters in our approach. In this estimate the profile shape effects have to be neglected and result in a scattering of the data around the logarithmic fit. The ITER reference point is found to be systematically below the fit of the extrapolated discharges, since the density profiles were assumed to be completely flat. The 0D figure of merit has to be chosen carefully, consistently with the specific set of assumption and with the parameter used as a performance meter. If this is the triple product, which is equivalent to $\mathrm{Q} /(\mathrm{Q}+5)$, then a scaling like $H \beta_{N, t h} / q_{95}^{2}$ is highly inaccurate in reproducing the simulations of the extrapolated discharges; in particular a systematic trend to overestimate the performance of high power discharges, which is inherent in its definition, is confirmed by the simulations presented here. For the three 


\section{Article: Extrapolation of ASDEX Upgrade H-mode discharges to ITER}

scaling laws considered here, choosing the correct figure of merit, the ASTRA predictions for $\mathrm{Q} /(\mathrm{Q}+5)$ are well aligned with the power laws. The scattering is less than $20 \%$ and it is shown to be due mainly to the density peaking, which improves the performance at a given $\beta_{N, t h}$, and to the variable power dependence of the fusion cross section on the ion temperature, which is a function of the ion temperature itself.

\section{References}

[1] ITER Physics Expert Group on Confinement and Transport, ITER Physics Expert Group on Confinement Modelling and Database and ITER Physics Basis Editors, Nuclear Fusion 39 (1999) 2175

[2] J. G. Cordey et al., Nuclear Fusion 45 (2005) 1078

[3] C. G. Petty et al., Fusion Sci. Technol. 43 (2003) 1

[4] D. C. McDonald et al., Plasma Phys. Control. Fusion 46 (2004) A215

[5] T. Takizuka et al., Plasma Phys. Control. Fusion 48 (2006) 799

[6] D. R. Mikkelsen et al., in Fusion Energy 1998 (Proc. 17th Int. Conf. Yokohama, 1998) (Vienna: IAEA) CD-ROM file ITERP1/08 and http://www.iaea.org/programmes/ripc/physics/start.htm

[7] V. Mukhovatov et al., Nuclear Fusion 43 (2003) 942

[8] G. V. Pereverzev, P. N. Yushmanov, IPP report 5/98 (2002)

[9] A. G. Peeters et al., Nuclear Fusion 47 (2007) 1341

[10] T. C. Luce et al., Physics of Plasmas 11 (2004) 2627

[11] D. E. Post, R. V. Jensen et al., Atomic Data and Nuclear Tables, 20 (1977) 397

[12] R. Neu et al., Fusion Technology 44 (2003), 692

[13] ITER Design Description Documents, N 11 DDD 178 04-06-04 R 0.4

[14] O. J. W. F. Kardaun et al., Nuclear Fusion 42 (2002) 841

[15] K. Borrass et al., Nuclear Fusion 44 (2004) 752

[16] Y. R. Martin et al., Journal of Physics, Conference Series 123 (2008), 012033 